Title: Driver response at tyre blow-out in heavy vehicles $\&$ the importance of scrub radius

Publisher: IEEE

DOI:10.1109/IVS.2014.6856544

Copyright: (C) 2014 IEEE. Personal use of this material is permitted. Permission from IEEE must be obtained for all other uses, in any current or future media, including reprinting/republishing this material for advertising or promotional purposes, creating new collective works, for resale or redistribution to servers or lists, or reuse of any copyrighted component of this work in other works.

Version: Accepted article

To cite this article: TAGESSON, Kristoffer; JACOBSON, Bengt; LAINE, Leo. Driver response at tyre blow-out in heavy vehicles \& the importance of scrub radius. In: Intelligent Vehicles Symposium Proceedings, 2014 IEEE. IEEE, 2014. p. 1157-1162.

To link to this article: http://dx.doi.org/10.1109/IVS.2014.6856544 


\title{
Driver Response at Tyre Blow-Out in Heavy Vehicles \& The Importance of Scrub Radius*
}

\author{
Kristoffer Tagesson ${ }^{1}$, Bengt Jacobson ${ }^{2}$ and Leo Laine ${ }^{1}$
}

\begin{abstract}
Front tyre blow-outs lead to several fatal accidents involving heavy vehicles. Common for most heavy vehicles is a positive scrub radius. This can result in a destabilising steering wheel torque at front tyre blow-out. In this study the safety improvement achieved when reducing scrub radius is quantified. By using a heavy truck equipped with a modified electric power steering system it was possible to change the scrub radius virtually. Brakes were configured to emulate front tyre blow-out which appeared as a sudden disturbance on one of the front tyres. In total 20 drivers took part in the study which was run on a test track at $50 \mathrm{~km} / \mathrm{h}$. Results show that the produced average lateral deviation from the original direction was $23 \mathrm{~cm}$, when scrub radius was $12 \mathrm{~cm}$, compared to $16 \mathrm{~cm}$, when scrub radius was $0 \mathrm{~cm}$. The main cause of the observed difference was a small, yet significant, initial overshoot in steering wheel angle which can be derived from the destabilising steering wheel torque.
\end{abstract}

\section{INTRODUCTION}

Tyre failures are involved in many fatal accidents every year. In [1] it was found that damaged tyres was the second most common vehicle defect reported at fatal accidents between 1995 and 1997 in USA. Blow-outs occurred in $0.35 \%$ of all fatal truck crashes. In particular front tyre blowouts seemed more critical than blow-outs on other axles. In [2] another sample was taken from a French motorway network of $2000 \mathrm{~km}$, during the period from 1996 to 2002. It showed that $3.5 \%$ of all trucks involved in accidents, with property damage or injury, were reported with blown out tyres. A higher criticality of front axle blow-outs was again confirmed.

Tyre blow-outs frequency can be reduced using correct tyre pressure and thereby avoid overheating. Overloading and excessive wear should also be avoided. Tyre pressure and loading monitoring systems have therefore been suggested and are already in use on many vehicles [1], [2]. A road hazard is another cause of tyre blow-outs. This problem is not removed by previously suggested countermeasures. In summary, it can be expected that the total number of tyre blow-outs on the roads will decline, yet a considerable number will remain. It is therefore of high importance to

\footnotetext{
*This work was supported by Volvo Trucks and Vinnova

${ }^{1}$ Kristoffer Tagesson and Leo Laine are with the Division of Vehicle Engineering \& Autonomous Systems, Chalmers University of Technology. They are also with Department of Chassis Strategies \& Vehicle Analysis, Volvo Group Trucks Technology, 40508 Gothenburg, Sweden kristoffer.tagesson@volvo. com respectively leo.lainedvolvo. com

${ }^{2}$ Bengt Jacobson is with the Division of Vehicle Engineering and $\mathrm{Au}-$ tonomous Systems, Chalmers University of Technology, 41296 Gothenburg, Sweden bengt. jacobson@chalmers. se
}

support drivers by designing vehicles that are insensitive to tyre blow-outs.

There are three different reasons for vehicle instability at front tyre blow-outs. Firstly, vehicle yaw torque is induced. A damaged tyre produces a lot higher rolling resistance than a normal tyre. At worst it even stops rolling and instead develops full slip, similar to a locked up tyre. Since this force is offset from vehicle centre a resulting torque around centre of gravity will be acting. Secondly, common for most heavy vehicles is a positive scrub radius, which is a consequence of wheel and axle geometry. This can result in a destabilising steering wheel, StW, torque during front tyre blow-out. A deflated tyre has a smaller radius than a normal tyre. This creates even higher scrub radius and consequently also higher StW torque [3]. Thirdly, a vehicle towing one or more trailers will experience forces in the connection point. E.g. if the towing vehicle is slowed down because of a blown out tyre a heavy towed trailer will create high forces in the connection point. If an angle has developed, between the units, this force will act destabilising on the towing vehicle. Combined, these effects can result in run of road, collision with oncoming vehicles, roll-over or jack-knife, unless the driver is able to balance the effects by steering or braking. When designing vehicles it is therefore important to know how a driver reacts at a tyre blow-out. More precisely put, it is important to understand driver behaviour as a function of all the three above mentioned instability factors. In this work we focus on driver behaviour in a heavy vehicle and try to distinguish between vehicle yaw torque and StW torque.

Many have studied and modelled the motion of cars and trucks at tyre blow-out, e.g. [4] and [5]. Few have studied the variety in behaviour among different drivers at tyre blowout. One exception is [6] where a truck simulator study was run. It was observed that driver behaviour was very much dependent on the effect of surprise. The lateral deviation on the first blow-out was a lot higher than on the following trials. The level of StW torque induced was however not varied.

To the best of the authors' knowledge no one has yet deeply analysed the influence of StW torque during tyre blow-out. In particular not for heavy vehicles where dynamics and steering geometries, e.g. scrub radius, are different than for cars. This will be the scope of this paper.

The outline of the paper is as follows: In section II the performed test track experiment is described and the corresponding results in section III. Finally some conclusions are given in section IV. Sign conventions used for vehicle quantities complies with ISO definitions, see [7]. 


\section{METHOD}

A test was set up with a 9 ton solo semi-trailer truck, commonly known as tractor unit, on a test track where 20 drivers were exposed to several repetitions of emulated front tyre blow-out. Research results were obtained through informed consent. The test was part of a larger program, e.g. see [8]. Drivers were not aware of the intention of the test, but had been exposed to three similar interventions prior to the blow-out runs, all pulling the vehicle left.

\section{A. Test Track}

The test was run on a closed test track in Sweden during two days in December. Temperature was $3-8^{\circ} \mathrm{C}$. The track was slightly wet, but it did not rain. For safety reason a $300 \mathrm{~m}$ long and $3.6 \mathrm{~m}$ wide straight marked lane on a large brake and handling area was used. This provided sufficient safety margins. To make drivers avoid crossing lane markings soft cones were put in the adjacent lanes. The set-up is illustrated in Fig. 1.

\section{B. Test Vehicle}

A solo $6 \times 2$ pusher tractor was used in the experiment having the pusher axle lifted. Brakes were controlled to emulate tyre blow-out. This was performed by applying $350 \mathrm{kPa}$ of brake pressure on one of the front tyres. This level was selected just below tyre locking. The produced tyre force was thereby nearly maximised, but discontinuities relating to ABS control was eliminated. The relatively high level was selected to produce worst case blow-out forces, which is still not far above what has been measured, e.g. see [4]. In the case that the driver pressed the brake pedal a select high pressure routine was used. If the driver pressed the accelerator pedal the test was aborted. Tyre dimensions were selected on purpose to get high scrub radius. This resulted in $12 \mathrm{~cm}$ which in the default set up produced around $3 \mathrm{Nm}$ of torque on the StW. For more details on the vehicle used see Table I.

The vehicle was also equipped with Volvo Dynamic Steering, which is an electric power steering unit. The system contains the ability to fully suppress steering torque disturbances coming from tyre road interaction, analogous

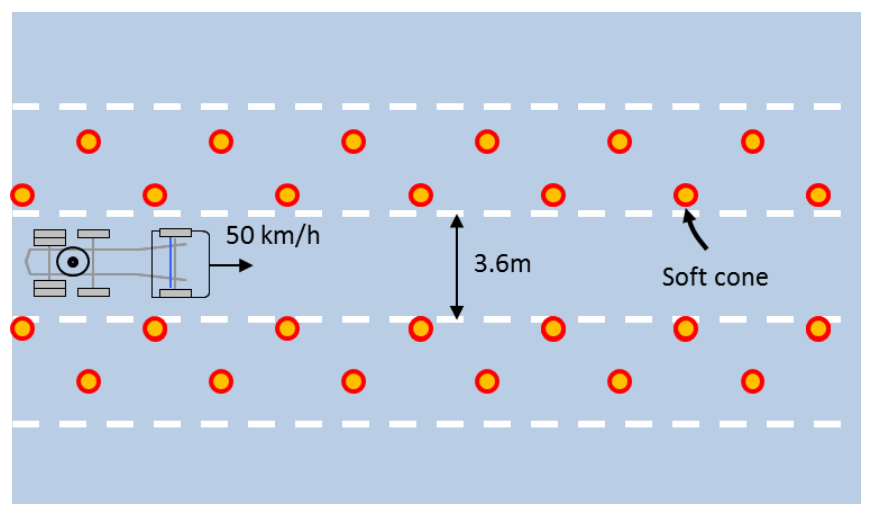

Fig. 1. Sketch of track set-up. Soft cones were used to create a sense of danger in the adjacent lanes.
TABLE I

SPECIFICATION OF VOLVO FH TRACTOR USED

\begin{tabular}{|c|c|c|c|}
\hline Property & Value & Unit & Description \\
\hline$L$ & 4.1 & $\mathrm{~m}$ & $\begin{array}{l}\text { Wheelbase, distance between front } \\
\text { and drive axle }\end{array}$ \\
\hline$F_{z, f}$ & 58470 & $\mathrm{~N}$ & Front axle vertical load \\
\hline$F_{z, p}$ & 0 & $\mathrm{~N}$ & Pusher axle vertical load (lifted) \\
\hline$F_{z, d}$ & 29430 & $\mathrm{~N}$ & Drive axle vertical load \\
\hline$i_{s}$ & 23.2 & - & $\begin{array}{l}\text { Steering ratio, road wheel angle to } \\
\text { StW angle }\end{array}$ \\
\hline$r_{S t W}$ & 0.225 & $\mathrm{~m}$ & $\begin{array}{l}\text { StW radius, measured from centre } \\
\text { to rim edge }\end{array}$ \\
\hline
\end{tabular}

to $0 \mathrm{~cm}$ of scrub radius in the blow-out case. The system was made configurable also to function as a conventional power steering system, however preserving the normal torque characteristics, which then is analogous to $12 \mathrm{~cm}$ of scrub radius. I.e. the two modes will behave the same during normal driving, but deviate when blow-out occurs. By changing mode in-between runs all drivers were exposed to tyre blowouts both with $0 \mathrm{~cm}$ and $12 \mathrm{~cm}$ of scrub radius.

The on-board truck sensors were recorded during the whole test. That includes e.g. yaw rate, lateral acceleration, StW angle, StW torque, wheel speeds, brake pressure, accelerator pedal position and brake pedal position. A high precision GPS, placed above the drive axle, was also used and recorded.

\section{Test Drivers}

In total 20 professional drivers took part, normally driving durability tests of trucks. Only one driver had experience from brake or handling tests. The average age was 43.5, the oldest participant was 63 and the youngest 27 . There were 17 male and 3 female.

\section{Test Procedure}

Drivers were told that the intention of the test was to record normal positioning in lane and that they should run back and forth inside the straight lane for $300 \mathrm{~m}$. Cruise control was set to $50 \mathrm{~km} / \mathrm{h}$. An operator fired off emulated tyre blow-outs on the front left wheel, as described, at random locations. At the same time cruise control was deactivated.

Each driver was exposed to three blow-outs per scrub radius. The order of the exposures was reversed for every new driver to avoid bias from learning. For some drivers an additional blow-out on the front right wheel was fired off.

\section{RESULTS AND ANALYSIS}

All trials have been checked with respect to; initial speed range $50 \pm 2 \mathrm{~km} / \mathrm{h}$, correct brake pressure, that the driver did not press the accelerator pedal, and that the brake pedal was not pressed hard. After this 103 front left blow-outs remain, where 51 are run with scrub radius $12 \mathrm{~cm}$ and 53 are run with scrub radius $0 \mathrm{~cm}$. In this series all drivers are represented in at least one run per scrub radius setting. Additionally, 15 front right blow-outs are also kept. 


\section{A. Left Blow-Out Path and Time Series}

Fig. 2 show all trajectories produced for front left blowout runs. Black colour is used for runs with $12 \mathrm{~cm}$ scrub radius. Red colour is used for runs with $0 \mathrm{~cm}$ scrub radius. Bold lines are used for average. The produced average lateral deviation from the original direction is $23 \mathrm{~cm}$, when scrub radius is $12 \mathrm{~cm}$, compared to $16 \mathrm{~cm}$ on average, when scrub radius is $0 \mathrm{~cm}$. There is however large variance in data, so a direct comparison will not prove a significant difference. Some drivers deviated left by more than $50 \mathrm{~cm}$.

Fig. 3 show time series of speed, StW angle, StW torque and yaw rate for all front left blow-out runs. Colouring used is the same as in Fig. 2. The speed profiles are as expected similar for all runs apart for some where the driver has pressed the brake pedal gently. The StW angle curves initially indicate that some drivers, exposed to a destabilising, StW torque turn left before they turn right. Furthermore during the first second the steering profile is rather consistent. After that, very different profiles appear. The StW torque curves show an apparent difference between the two settings used.

Continuing on analysing Fig. 3, it can be seen that the yaw rate response roughly show a one period sine wave. Corresponding frequency, $0.7 \mathrm{~Hz}$, happens to match the resonance frequency of several truck combination types, see [9]. This highlights the importance of extending the study for multi-unit truck combinations.

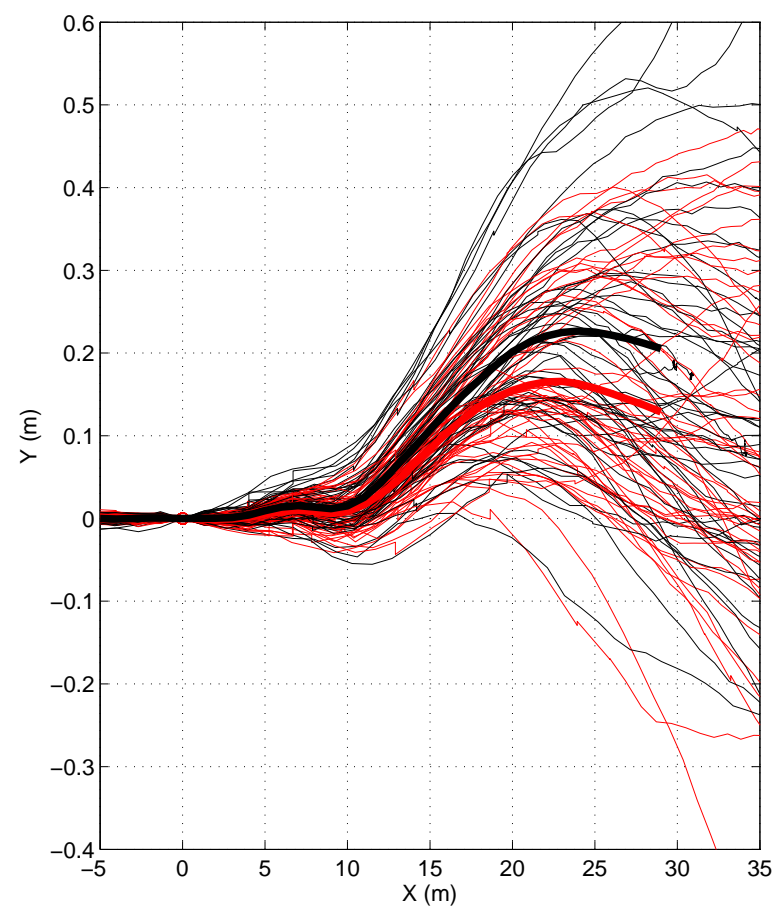

Fig. 2. Trajectories of centre of drive axle for all emulated front left blow-out runs. The curves have been rotated and moved so that blowout is initiated at position $(0,0) \mathrm{m}$ running at zero heading. Thin red lines correspond to scrub radius $0 \mathrm{~cm}$. Thin black lines correspond to scrub radius $12 \mathrm{~cm}$. Bold red line correspond to average of scrub radius $0 \mathrm{~cm}$ runs. Bold black line correspond to average of scrub radius $12 \mathrm{~cm}$ runs.
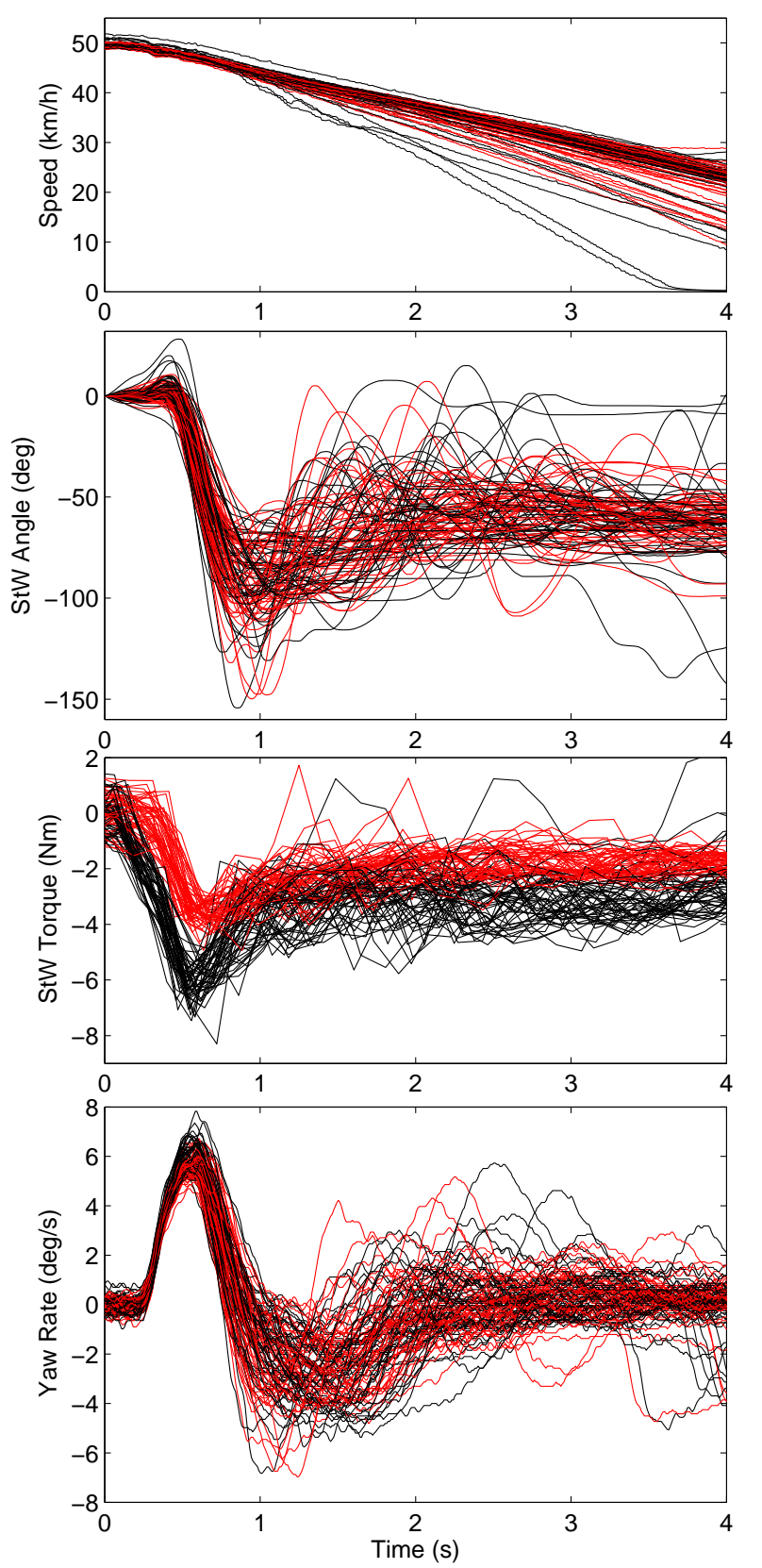

Fig. 3. Time series for all emulated tyre blow-out runs. The blow-out is initiated at time $0 \mathrm{~s}$. Red lines correspond to scrub radius $0 \mathrm{~cm}$. Black lines correspond to scrub radius $12 \mathrm{~cm}$. In the first subfigure drive axle wheel speed is shown. The second subfigure show StW angle which is adjusted to 0 deg at time zero. The third subfigure show StW torque. The last subfigure show yaw rate.

In general, drivers that got low lateral deviation responded early and used high StW angle rate.

\section{B. Statistical Analysis of Scrub Radius Settings}

Trajectories, seen in Fig. 2, StW angle and yaw rate, seen in Fig. 3, indicate a difference when scrub radius was changed. The variance is however so high that this difference is not significant when the two groups are treated as independent, but drivers in the two groups are actually not independent. The same drivers have been used in both 
groups. Therefore we can use a paired difference test to analyse the relative change for each driver. By doing so the variance used when comparing the groups will be scaled by $1 / n$, where $n$ is the number of drivers, in this case 20 . The two groups will hereafter be denoted as the $12 \mathrm{~cm}$ and the $0 \mathrm{~cm}$ group respectively.

In Fig. 4 a paired t-test is performed on the travelled path data from left tyre blow-outs. First, the average path is calculated for each driver, with the two groups kept apart. Then, for each driver, the average path from the $12 \mathrm{~cm}$ runs is subtracted from the $0 \mathrm{~cm}$ runs. This is shown in black in the first subfigure. In other words it is the measured reduction in lateral deviation for each driver achieved when lowering the scrub radius. The average of these 20 curves is shown in bold red. After $24 \mathrm{~m}$ of longitudinal displacement the average improvement is $6.4 \pm 4.4 \mathrm{~cm}$, using a $95 \%$ confidence interval. $24 \mathrm{~m}$ is also where the maximum average displacement is observed in Fig. 2. Fig. 4 also include t-value with 19 degrees of freedom. To test if the average reduction is significant a two-tailed t-value with $98 \%$ confidence is used. This gives a t-value threshold of 2.54 which is also marked in the graph (for $99 \%$ confidence level the value is 2.86 ). The $98 \%$ confidence limit is surpassed after $15 \mathrm{~m}$ of longitudinal displacement. The highest t-value, 3.14, is reached after $21 \mathrm{~m}$. It can therefore be concluded with confidence that drivers are affected by the StW torque they are subjected to. Also that the lateral deviation is lowered by having a lower scrub radius, or as in the case of the tested vehicle a power steering system that eliminates disturbances.

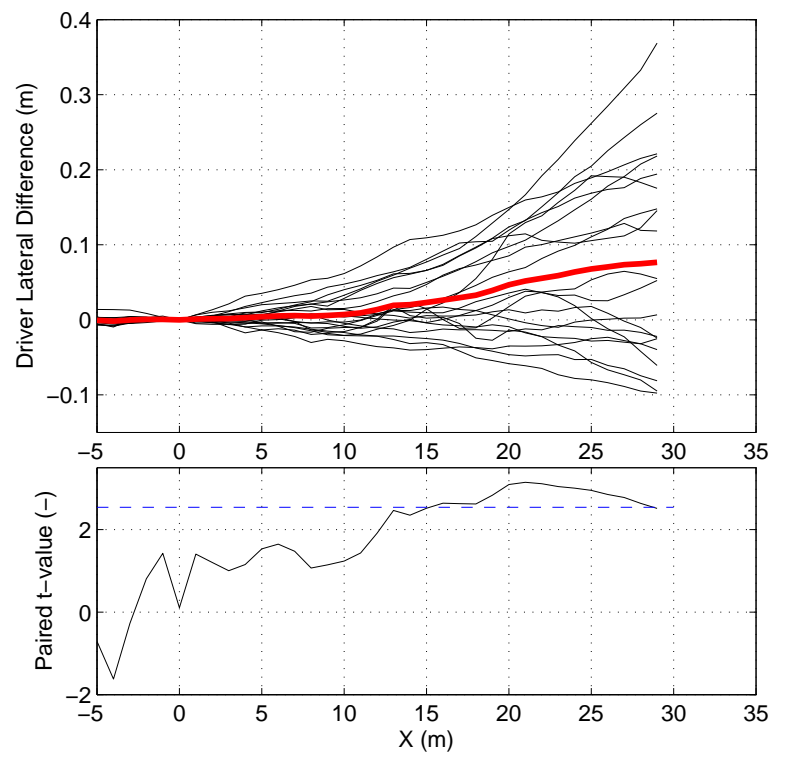

Fig. 4. A paired t-test of trajectories. In black the first subfigure show average lateral difference per driver between runs with $0 \mathrm{~cm}$ scrub radius and $12 \mathrm{~cm}$ scrub radius. The red bold curve is the average of all drivers. The second subfigure show the corresponding t-value, in solid black. Also included is a dashed blue line at 2.54 which is equal to $\mathrm{t}_{19,0.99}$, i.e. the two-sided $98 \%$ cumulative probability value for 19 degrees of freedom.

To get a better understanding of the cause of the improvement identified we perform the same paired test also for
StW angle and yaw rate. The result is shown in Fig. 5 and Fig. 6 respectively. For StW angle we can now prove the significance for the groups between $0.3 \mathrm{~s}$ and $0.5 \mathrm{~s}$. Drivers running with scrub radius $12 \mathrm{~cm}$ are here pulled by the disturbing StW torque in the wrong direction before they react and actively start to balance the blow-out by steering. However it should be noted that the significance is not strong. When using 99\% confidence level the difference would not prove significant. For yaw rate a difference is also observed. Here the significance is stronger. A rough estimate show that the observed difference in StW angle is large enough to cause the observed difference in yaw rate. And the observed difference in yaw rate is large enough to cause the difference in lateral displacement.

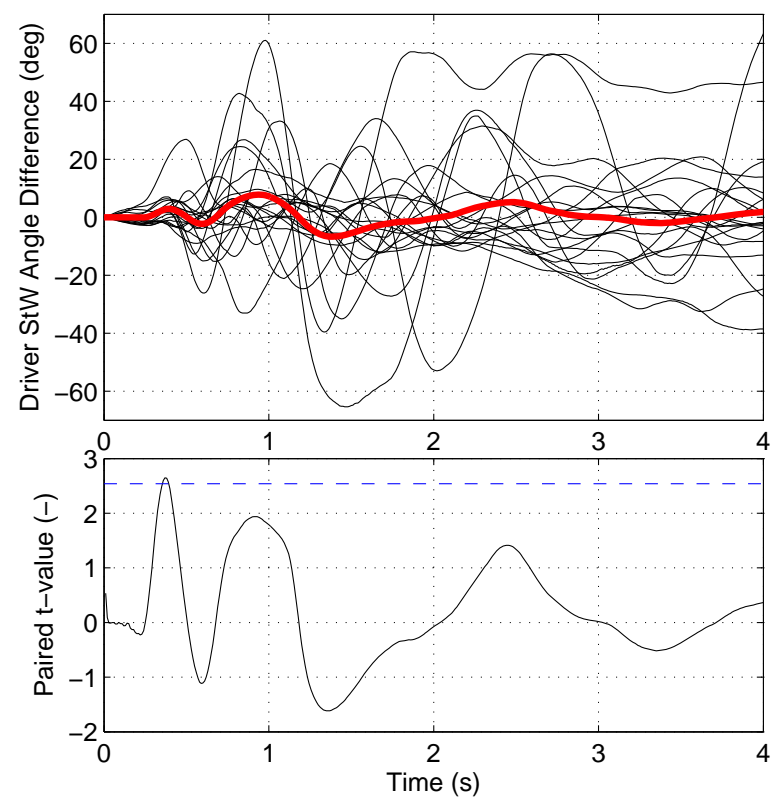

Fig. 5. A paired t-test of StW angle. In black the first subfigure show average $\mathrm{StW}$ angle difference per driver between runs with $0 \mathrm{~cm}$ scrub radius and $12 \mathrm{~cm}$ scrub radius. The red bold curve is the average of all drivers. The second subfigure show the corresponding t-value, in solid black. Also included is a dashed blue line at 2.54 which is equal to $t_{19,0.99}$, i.e. the two-sided $98 \%$ cumulative probability value for 19 degrees of freedom.

\section{Open Loop Response}

Fig. 7 show all trajectories, just like Fig. 4, but here a dashed green line is also included to show the open loop vehicle response. I.e. a run where $\mathrm{StW}$ angle was locked at $0 \mathrm{deg}$. For this run the lateral deviation quickly becomes more than a lane. The importance of having an alert driver is obvious.

\section{Subjective Comparison of Scrub Radius}

The virtual change made of scrub radius between the first and the last trials was kept secret to the drivers. Directly after the last run all drivers were asked if they had experienced any difference. Some reported that they had perceived the disturbance at blow-out as higher in some of the runs compared to others. Objectively, the level of the disturbance was the same 


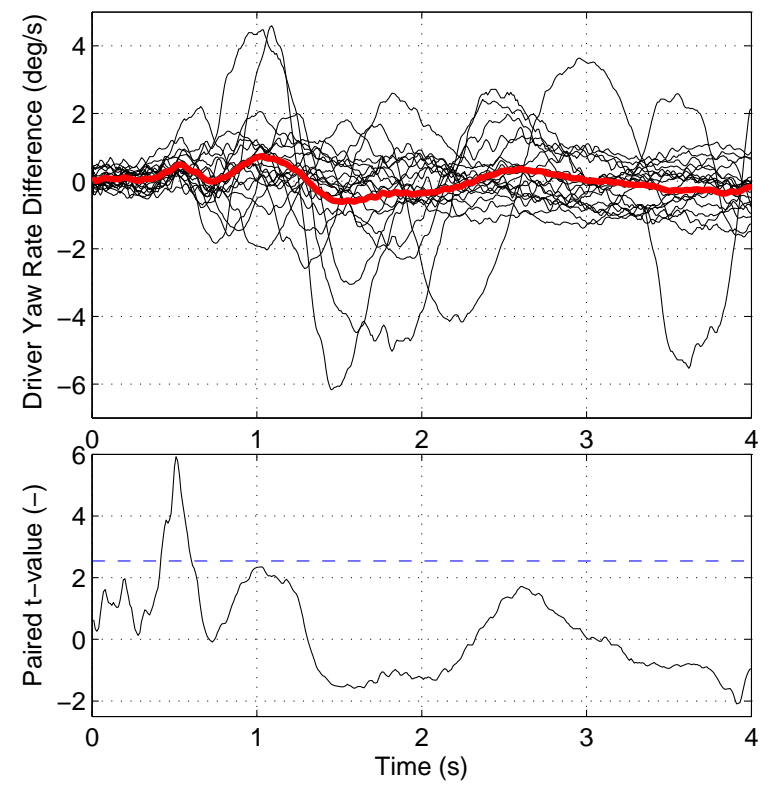

Fig. 6. A paired t-test of yaw rate. In black the first subfigure show average $\mathrm{StW}$ angle difference per driver between runs with $0 \mathrm{~cm}$ scrub radius and $12 \mathrm{~cm}$ scrub radius. The red bold curve is the average of all drivers. The second subfigure show the corresponding t-value, in solid black. Also included is a dashed blue line at 2.54 which is equal to $t_{19,0.99}$, i.e. the two-sided $98 \%$ cumulative probability value for 19 degrees of freedom.

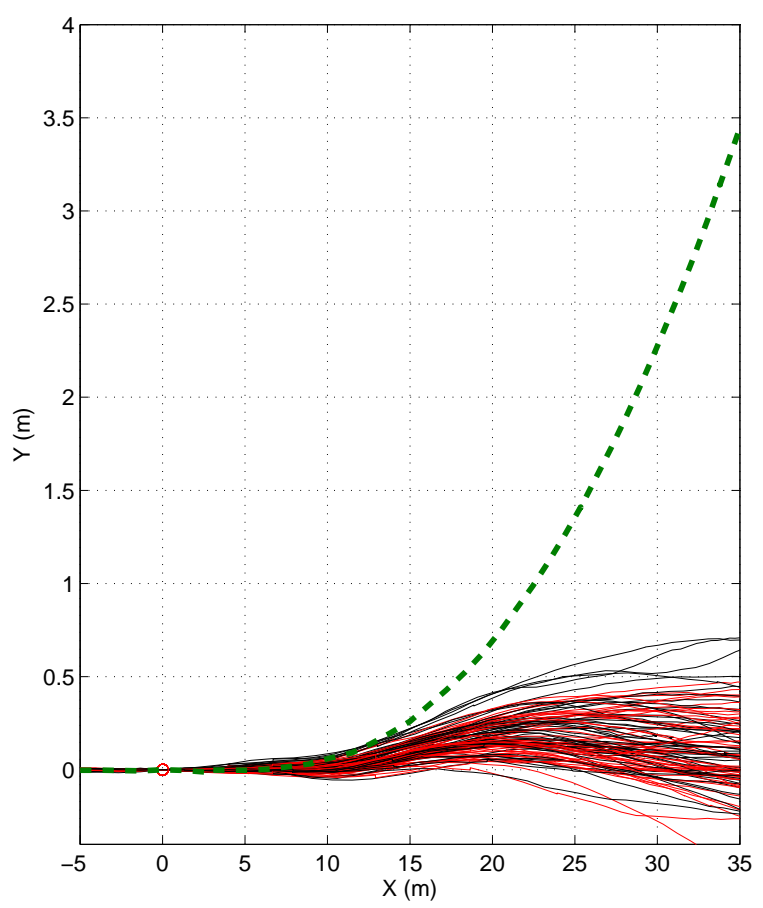

Fig. 7. Trajectories for all emulated left right blow-out runs in comparison to an open loop response. The curves have been rotated and moved so that blow-out is initiated at position $(0,0) \mathrm{m}$ running at zero heading. Thin red lines correspond to scrub radius $0 \mathrm{~cm}$. Thin black lines correspond to scrub radius $12 \mathrm{~cm}$. Bold dashed green line correspond to the open loop response, i.e. StW angle locked at $0 \mathrm{deg}$. for all runs. No one reported that they had felt a difference connected to steering. As a follow-up question, all drivers were also asked if they had experienced any difference in the steering system. No one had. In Fig. 3 the difference in terms of steering wheel torque is apparent between the two settings. About $3 \mathrm{Nm}$ of disturbance reaches the driver when scrub radius is $12 \mathrm{~cm}$. As a separate experiment $3 \mathrm{Nm}$ was applied to the StW during normal driving for a few drivers. All noticed that a disturbance had been applied. The difference between the normal and the critical situation is obviously an example of how the mental ability, to perform concurrent tasks, is dependent on the intensity of the main task.

\section{E. Right Blow-Out}

Some drivers were also exposed to a blow-out on the right front wheel directly after the main series of blowouts on the front left wheel. Fig. 8 show trajectories for these runs. The average lateral deviation produced increased compared to left-blow out runs. Drivers had become used to a disturbance on the left front wheel. In line with [6] this show that repeated exposures will reduce the lateral deviation, as the driver focus harder and learn the manoeuvre.

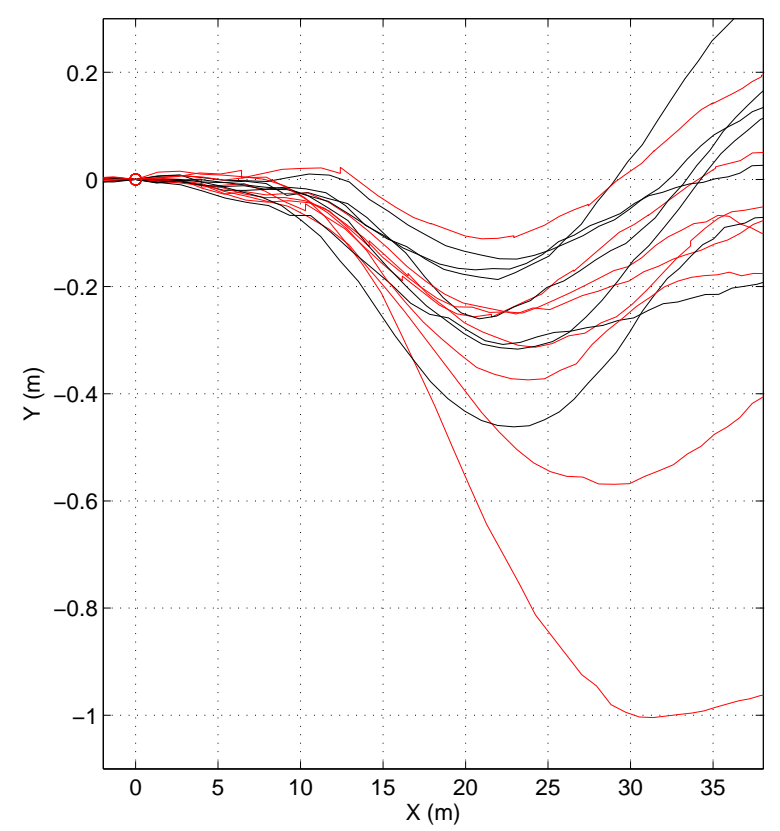

Fig. 8. Trajectories for all emulated front right blow-out runs. The curves have been rotated and moved so that blow-out is initiated at position $(0,0) \mathrm{m}$ running at zero heading. Thin red lines correspond to scrub radius $0 \mathrm{~cm}$. Thin black lines correspond to scrub radius $12 \mathrm{~cm}$.

\section{CONCLUSION}

A test was set up with a 9 ton solo tractor on a test track where 20 drivers were exposed to repeated exposures of emulated worst case front tyre blow-outs. By using a configurable power steering system it was possible to alter 
between two scrub radius settings, one corresponding to $12 \mathrm{~cm}$ and one to $0 \mathrm{~cm}$. It was observed that the lateral deviation produced at a blow-out was lowered by $6.4 \pm 4.4 \mathrm{~cm}$ when scrub radius was changed from $12 \mathrm{~cm}$ to $0 \mathrm{~cm}$. The difference would increase for drivers holding the StW loose. In particularly, the improvement for drivers not holding the StW at all would be several meters. Low scrub radius or a power steering system, that removes disturbances, could therefore ultimately slightly reduce the number of fatalities caused by tyre blow-outs.

Results also reveal that the response received from different drivers vary widely at a blow-out, irrespective of scrub radius setting. This is for instance reflected in reaction time and steering rate. These two measures have been identified as very important as to be able to maintain low lateral deviation. The test was set up with drivers that knew they would be exposed to some sort of challenge. As seen in [6] it is therefore most likely that e.g. reaction time would be higher under normal circumstances, as also partly was confirmed with results from right front tyre blow-outs. On average there is a statistically significant improvement of lowering scrub radius, but for an inattentive and less skilled driver yet more support would be needed to secure all scenarios.

To further reduce the number of accidents involving defective tyres there are several additional solutions that can be developed. First observation, reaction time is obviously critical. Designing tyres that always deflate slowly at the event of failure would therefore be beneficial. Developing stability support using brakes or additional steering, to reduce the initial heading error, would be another method. Using stabilising steering torque could be a third way. Next observation, when considering a vehicle combination with more than one unit the dynamics of the full vehicle must be considered. Here knowledge about driver behaviour is missing. When this information is available all previously suggested methods may need adjustments. And final observation, all drivers being part of this study had improved their deviation when exposed to a blow-out by the end of the session. Practical training should not be underestimated.

\section{REFERENCES}

[1] Z. Bareket, D. F. Blower, and C. MacAdam, "Blowout resistant tire study for commercial highway vehicles," The University of Michigan, Tech. Rep., 2000.

[2] J.-L. Martin and B. Laumon, "Tire blow-outs and motorway accidents." Traffic injury prevention, vol. 6, no. 1, pp. 53-5, Mar. 2005. [Online]. Available: http://www.ncbi.nlm.nih.gov/pubmed/15823875

[3] S. Patwardhan and M. Tomizuka, "Theory and experiments of tire blow-out effects and hazard reduction control for automated vehicle lateral control system," in American Control Conference, Baltimore, 1994, pp. 7-9. [Online]. Available: http://ieeexplore.ieee.org/xpls/ abs_all.jsp?arnumber $=752248$

[4] K. Chakravarthy, "Development of a steer axle tire blowout model for tractor semitrailer in Trucksim," 2013. [Online]. Available: http://rave.ohiolink.edu/etdc/view?acc_num=osu1367533852

[5] W. Blythe, T. Day, and W. Grimes, "3-dimensional simulation of vehicle response to tire blow-outs," in SAE International Congress and Exposition, vol. 1998, no. 724, Detroit, Feb. 1998. [Online]. Available: http://trid.trb.org/view.aspx?id=499586

[6] H. E. Pettersson, J. Aurell, and S. Nordmark, "Truck driver behaviour in critical situations and the impact of surprise," in DSC 2006 Europe, Paris, 2006.
[7] ISO, "International standard 8855 , Road vehicles and road holding ability - Vocavulary," 1991.

[8] K. Tagesson, B. Jacobson, and L. Laine, "Driver response to automatic braking under split friction conditions," in Submitted to AVEC, Tokyo, 2014.

[9] S. Kharrazi, "Steering based lateral performance control of long heavy vehicle combinations," Ph.D. dissertation, Chalmers University of Technology, 2012. 